\title{
The History of Nuclidic Masses and of their Evaluation
}

\author{
Georges Audi \\ Centre de Spectrométrie Nucléaire et de Spectrométrie de Masse, CSNSM, \\ IN2P3-CNRS, et UPS, Bâtiment 108, F-91405 Orsay Campus, France \\ Contribution to the special issue of \\ the "International Journal of Mass Spectrometry" (IJMS) \\ in the honor of the 65th anniversary of Jürgen Kluge's birthday \\ (submitted December 24, 2005, resubmitted January 27, 2006)
}

\begin{abstract}
This paper is centered on some historical aspects of nuclear masses, and their relations to major discoveries. Besides nuclear reactions and decays, the heart of mass measurements lies in mass spectrometry, the early history of which will be reviewed first. I shall then give a short history of the mass unit which has not always been defined as one twelfth of the carbon-12 mass. When combining inertial masses from mass spectrometry with energy differences obtained in reactions and decays, the conversion factor between the two is essential. The history of the evaluation of the nuclear masses (actually atomic masses) is only slightly younger than that of the mass measurements themselves. In their modern form, mass evaluations can be traced back to 1955. Prior to 1955, several tables were established, the oldest one in 1935 .
\end{abstract}

PACS. 01.40.Di ; 01.65.+g ; 06.20.Dk ; 06.20.Fn ; 07.75.+h ; 21.10.Dr ; 23.40.-s ;23.50.+z;23.60.+e

Keywords: Nuclear binding energies - atomic masses - history of atomic masses - history of mass spectrometry - evaluation of atomic masses

\section{The history of nuclear masses}

The history of nuclear masses is almost as old as that of nuclear physics itself. It started with the development of mass spectrography in the late 1910's ${ }^{1}$. Mass spectrography itself was born in 1898 from the works of Wilhelm Wien. He analyzed, with a magnet, the so-called 'channel rays' ${ }^{2}$ discovered 12 years earlier by Eugen Goldstein.

In the following, I shall give the important steps in the early history of mass spectrometry with special focus on nuclear masses. The guideline will be given by the discoveries in physics that thrived on them, rather than by the techniques or results for themselves.

\footnotetext{
${ }^{1}$ Writing about history is a particular exercise, not straightforward for scientists. Stating "The history of nuclear masses is as old as nuclear physics" depends of course on definition. A.H. Wapstra remarked that: "One could argue that it started in 1869 when Mendeleiev published the periodical system of elements, in which (average) atomic masses were the basis." I hope that, in this historical sketch, I have not deviated too far from the truth.

${ }^{2}$ or 'kanalstrahlen', the stream of positive ions formed from residual gases in cathode ray tubes.
} 


\section{First mass spectrographs}

In 1907, Joseph John Thomson ${ }^{3}$ built a spectrograph with aligned magnetic and electric fields, having ions of the same species focused on the photographic plate along parabolas, see Fig.1] The resolving power of this spectrograph was around $R=10-20$. In

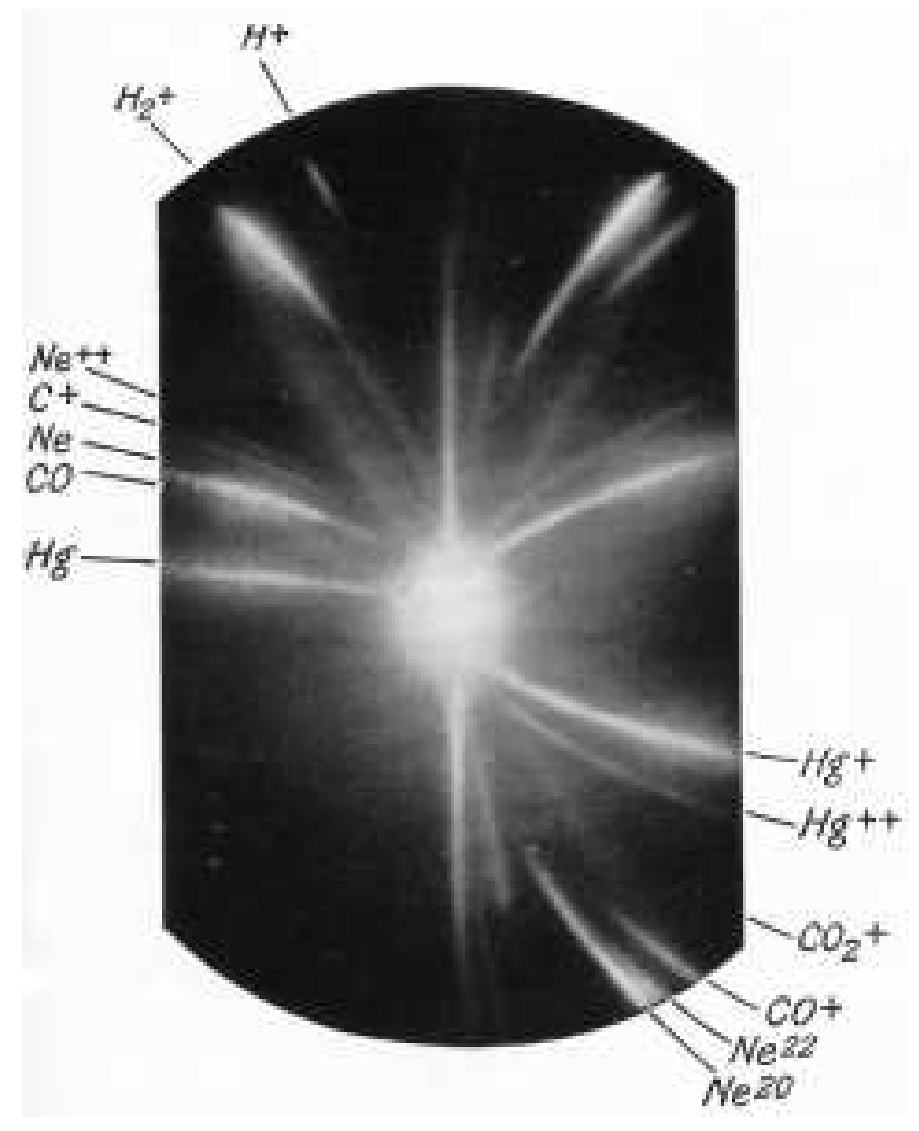

Figure 1: Photographic plate of the Thomson spectrograph. On this picture $\vec{B}$ and $\vec{E}$ are aligned along the horizontal axis $O x$. Ions with the same mass will have positions $x=k_{E} \times q / m v^{2}$ and $y=k_{B} \times q / m v$, then $x=\frac{k_{E}}{k_{B}^{2}} \times \frac{m}{q} \times y^{2}$ independently of their velocity $v$. They lie thus along a parabola. From Ref. [1].

1912, he obtained mass spectra of several gas compounds: $\mathrm{N}_{2}, \mathrm{O}_{2}, \mathrm{CO}, \mathrm{CO}_{2}, \ldots$ and was able to observe negatively-charged and also multiply-charged ions. One year later (1913) he made one of the most important discoveries in nuclear physics; he observed neon at two very different masses, $A=20$ and $A=22$. This was the discovery of "isotopism" 4 from direct observation of two different nuclidic species for the same element.

\footnotetext{
${ }^{3}$ J.J. Thomson was known already for his discovery of the electron in 1897, when he found that cathode rays were made of negative charged particles. He later built the plum-pudding model. An experiment of his former student, Ernest Rutherford, in 1911, showed that this model was not right.

${ }^{4}$ Frederick Soddy was the first to use the word "isotope". He discovered, in 1910, that the average mass of natural lead (that we know today to be a mixture of four isotopes), and of lead obtained in the decay of uranium or of thorium differed beyond possible experimental uncertainties. He considered
} 
Then, a long series of improvements followed which increased the resolving power and the sensitivity of the mass spectrographs and mass spectrometers ${ }^{5}$.

The first of these improvements was introduced by Arthur Jeffrey Dempster, at the University of Chicago, who, in 1918, built the first mass spectrometer. Low-energy ions were accelerated to high energy (500 to 1750 volts) and deflected by a constant magnetic field. They were thus almost mono-energetic. A resolving power of $R=100$ was achieved by this spectrometer ${ }^{6}$.

In 1919 Francis William Aston, who was J.J. Thomson's graduate student at Cambridge, built an instrument that was able to focus ions of the same species, independently of their velocity spread (energy focusing). This increased the resolving power of his spectrograph up to $R=130$. He thus obtained relative precisions of $10^{-3}$ in mass measurements ${ }^{7}$ with his first apparatus, see Fig.2

With this limited precision he obtained two of the most remarkable results. First, he was able to restore the "whole number rule": all masses (except hydrogen, see below) are whole numbers (which is true at this level of $10^{-3}$ ), and a fractional 'chemical' mass, like 35.5 for chlorine, is in reality a mixture of the two isotopes at $A=35$ and 37 with ratios $3 / 4$ and $1 / 4$. The second remarkable result, and probably one of the most important discovery for the story and the evolution of the Sun and the solar system, is that hydrogen is an exception, with a mass of 1.008 (as always, based on ${ }^{16} \mathrm{O}=16$ ). And this value "agrees with the value accepted by chemists" [2]. It was the theoretician and astronomer Arthur Stanley Eddington, searching for a way out of the scientific crisis concerning the age of the Sun, who immediately gave the answer. He calculated [3] that the unaccounted energy necessary for the extraordinary long lifespan of the Sun was due to "sub-atomic energy": "There is sufficient (energy) in the Sun to maintain its output of heat for 15 billion years. ... Aston has further shown conclusively that the mass of the helium atom is less than the sum of the masses of the 4 hydrogen atoms which enter into it... There is a loss of mass in the synthesis amounting to about 1 part in 120, the atomic weight of hydrogen being 1.008 and that of helium just 4... We can therefore at once calculate the quantity of energy liberated when helium is made out of hydrogen. If 5 per cent of a star's mass consists initially of hydrogen atoms, which

these "isotopes" to be a peculiarity of radioactive materials: their nature was not understood at that time as due to different nuclidic species.

${ }^{5}$ The detector in a mass spectrograph is a photographic plate where ions of different masses strike at different locations. Whereas in mass spectrometers a spectrum is constructed by varying a parameter responsible for the acceleration or the deflection of the ions. The variations of ionic current at a fixed position is then recorded by means of an electrometer. The advantage of the photographic plate is its ability to simultaneously record lines corresponding to various ionic species. Also, in earlier times, the precision in the position of the lines was twice better. Only much later (see below) will the precision in the position of an electronically recorded peak gain several order of magnitudes. F.W. Aston in Ref. [1], p. 38: " . . the word 'mass spectrograph' has lost the original restrictions intended by the writer when he introduced it in 1920, and is now loosely applied to any method of positive ray analysis, even, by a quite unnecessary anachronism, to the parabola method. It is best restricted to those forms of apparatus capable of producing a focused mass spectrum of lines on a photographic plate. An apparatus in which the focused beam of rays is brought up to a fixed slit, and there detected and measured electrically is best termed a 'mass spectrometer'. The first of these was devised by Dempster ... though the term was not introduced till much later."

${ }^{6}$ Dempster's second apparatus, in 1922, reached $R=160$ and precisions of $6 \times 10^{-4}$.

${ }^{7}$ J.-L. Costa, in 1925, built in Paris, an improved similar spectrograph and gained a factor two in resolving power and a precision of $3 \times 10^{-4}$. 


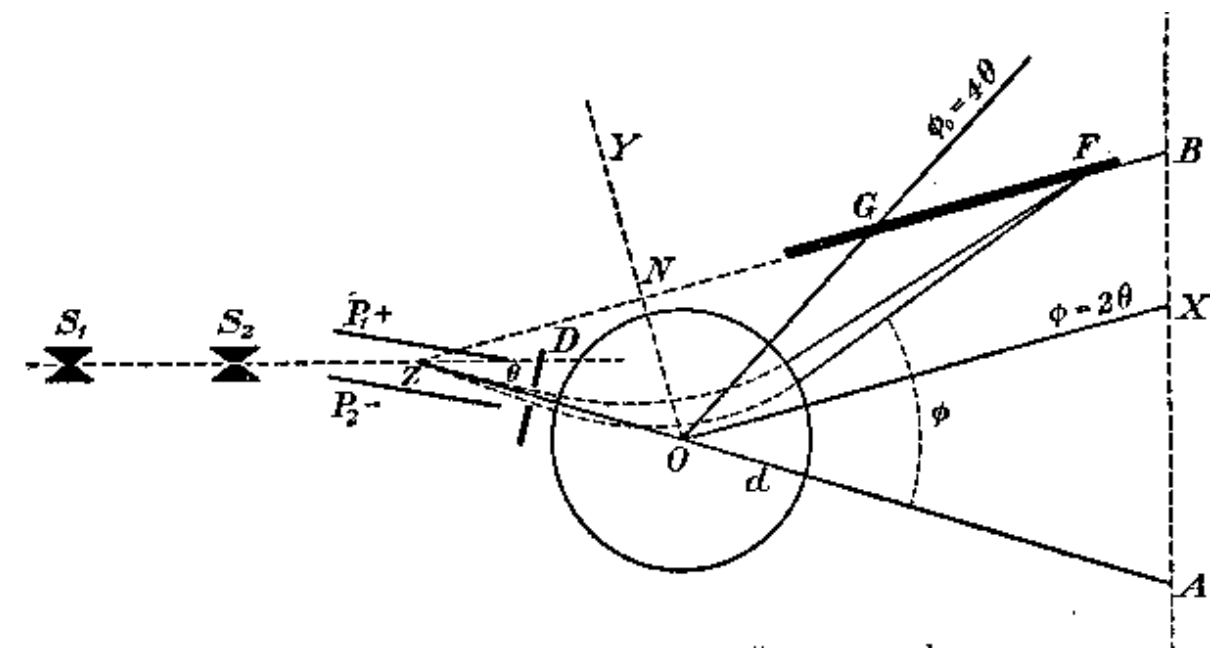

Figure 2: Diagram of Aston's first mass spectrograph (1919). The narrow slits $S_{1}$ and $S_{2}$ define a beam with very small divergence. They are deflected and dispersed by the electric field between $P_{1}$ and $P_{2}$. The diaphragm $D$ selects ions in a small window in energy which enter the magnetic field and are refocused at $F$ on the photographic plate $G F$. From Ref. 1].

are gradually being combined to form more complex elements, the total heat liberated will more than suffice for our demands, and we need look no further for the source of a star's energy."

With his second spectrograph, built in 1925, Aston achieved a resolving power of $R=600$ and could thus perform mass measurements with a relative precision of $10^{-4}$. He could then observe that the actual masses were shifted by some $8 \times 10^{-4}$ from the positions corresponding to full numbers, discovering thus, in 1927, the "mass defect" 8 , see Fig. 3. (Aston built a third instrument in 1937 and reached $R=2000$ ).

The ensemble of masses obtained by Aston were determinant in the discovery of closed shells by Walter M. Elsasser in Paris in 1933: $Z=2$ and 8 (corresponding to mass numbers 4 and 16) in Ref. 4]-a, see Fig. [4 $Z=20,28$ and 50 in Ref. 4]-b. Elsasser also showed, in an independent study, that $Z=82$ and $N=126$ correspond to shell closures based on the alpha decay energies, see Ref. [5]. "One of the main nuclear features which led to the development of the shell structure is the existence of what are usually called the magic numbers. That such numbers exist was first remarked by Elsasser in 1933." 6]. Strangely enough, the concept of shells was dropped by the physicists of that time who were satisfied with the liquid drop model, which seemed to efficiently describe fission. Shells were rediscovered 14 years later, in 1948, by Maria Goeppert-Mayer [7] when she examined a variety of observables, including Elsasser's publications, looking closely at the systematics of binding energies. The complete set of arguments, none of them individually conclusive in her opinion, was convincing enough

\footnotetext{
${ }^{8}$ Aston called the shift "packing fraction" defined as the percentage of deviation of the masses from whole numbers (choosing ${ }^{16} \mathrm{O}=16$, a convention established by Aston and that lasted until 1960, see below) expressed in parts per 10000 .
} 


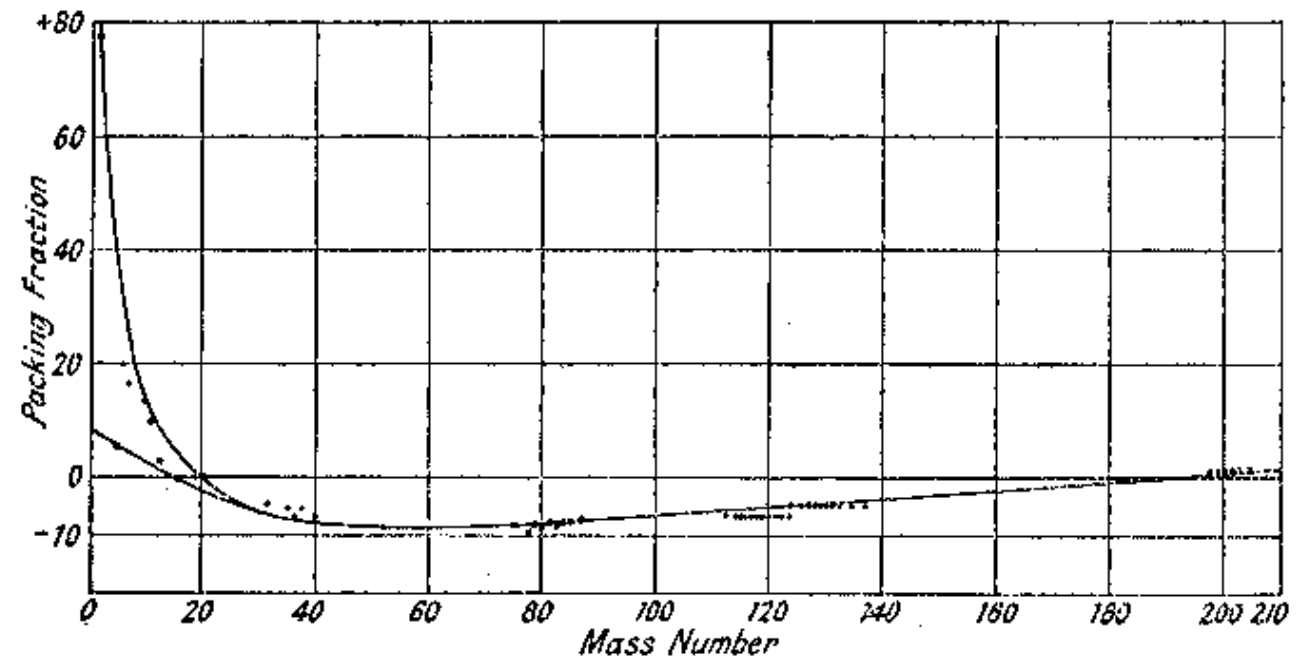

Figure 3: Aston's original packing fraction curve (1927). $P=10000 \times \frac{\mathcal{M}-A}{A}$, where $\mathcal{M}$ is the atomic mass and $A$ the mass number. From Ref. 1].

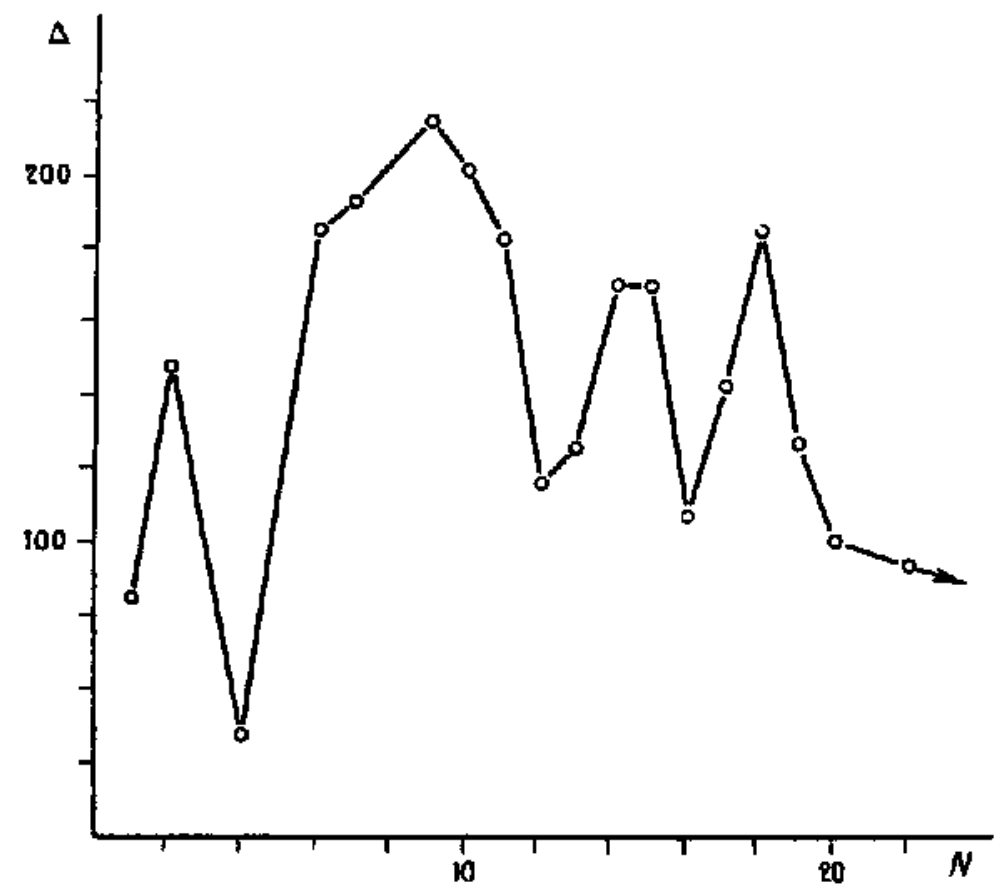

Figure 4: Plot of $\Delta=$ Mass $-N \frac{35.9760}{36}$, where $N$ is here the mass number. Clear minima of masses (maxima in binding energy) are evidenced at mass numbers 4 and 16, i.e. for ${ }^{4} \mathrm{He}$ and ${ }^{16} \mathrm{O}$. From Ref. [4-a. 
to claim the existence of "magic numbers" [] at $N$ (or $Z$ ) $=20,50,82$ and 126 . Magic numbers are much better demonstrated, nowadays, in the sudden decrease in the separation energies ${ }^{10}$ after these numbers, similar to the ionization potential after filling each atomic shell and responsible for the table of Mendeleiev.

In 1932, Kenneth T. Bainbridge combined a Wien-type velocity filter to a semicircular magnet spectrograph and reached a resolving power of $R=600$ and a $10^{-4}$ relative precision on masses. The measurements he performed allowed him to verify experimentally the equivalence of mass and energy. F.W. Aston in Ref. 1] p. 85, says about K.T. Bainbridge: "By establishing accurate comparisons of the masses of the light particles concerned in nuclear disintegrations, particularly that of ${ }^{7} \mathrm{Li}$, discovered by Cockcroft and Walton, he achieved a noteworthy triumph in the experimental proof of the fundamental theory of Einstein of the equivalence of mass and energy."

\section{Double-focusing spectrographs}

The general idea then, in the early 30's, was to build a spectrograph that would focus not only in velocity (energy) but also in direction: the "double-focusing" mass spectrograph. In a very short time interval, both concepts and designs flourished. Richard F.K. Herzog developed the theory of focusing in 1934. Then, simultaneously, and independently, three apparatuses were built: one by Arthur J. Dempster ${ }^{11}$ in Chicago, in 1935, yielding a resolving power of $R=3000$, see Fig. Kenneth T. Bainbridge and Edward B. Jordan, at Harvard, in 1936, with a different geometry, achieving $R=10000$ and a mass precision of $10^{-5}$, see Fig. by Josef H.E. Mattauch and Richard F.K. Herzog, in Vienna, in 1936, with a resolving power of $R=6000$, for their first spectrograph ${ }^{13}$.

Mass measurements with a precision of $10^{-5}$ were routinely achieved then. The most remarkable result obtained by Dempster in 1938, only 3 years after commissioning his spectrograph, was a greatly improved "packing fraction" curve, see Fig.6] that exhibited structures not seen in Aston's (Fig. 3). It is interesting to compare this 1938 curve to the "modern" one of Duckworth of 1958, Fig.7.

Alfred O. Nier adopted, in the late 40's, the mass spectrometry's detection technique (for the first time after the early Dempster's 1918 spectrometer, see above). With his first double-focusing device, he obtained a resolving power of $R=14000$; and, with his

\footnotetext{
${ }^{9}$ It was actually Eugene Paul Wigner who coined the term "magic number". The physicists community at that time favored the liquid-drop model. "Wigner too believed in the liquid drop model, but he recognized, from the work of Maria Mayer, the very strong evidence for the closed shells. It seemed a little like magic to him, and that is how the words 'Magic Numbers' were coined.", said Steven A. Moszkowski, who was a student of Maria Goeppert-Mayer, in a talk presented at the APS meeting in Indianapolis, May 4, 1996. The rediscovery of "magic numbers", lead M. Goeppert-Mayer herself, and independently J.Hans D. Jensen in Europe, one year later in 1949, to the construction of the shell model with strong spin-orbit coupling, and to the Nobel prize they shared with Wigner in 1963.

${ }^{10}$ see, e.g. $S_{2 n}$ and $S_{2 p}$ graphs in [8], p. 542, and more graphs on the AmDC web site 9].

${ }^{11}$ R.C. Barber comments [10]: "Following the first instruments, there were several that were developed with partial focusing based on simple geometric ideas. The development of the theory of focusing, by Herzog in 1934, gave rise to a generation of instruments in the late 30's that made use of the new insights."

${ }^{12}$ four years later E.B. Jordan reached $R=30000$.

${ }^{13}$ several devices followed, built along the same line, yielding as much as $R=100000$.
} 

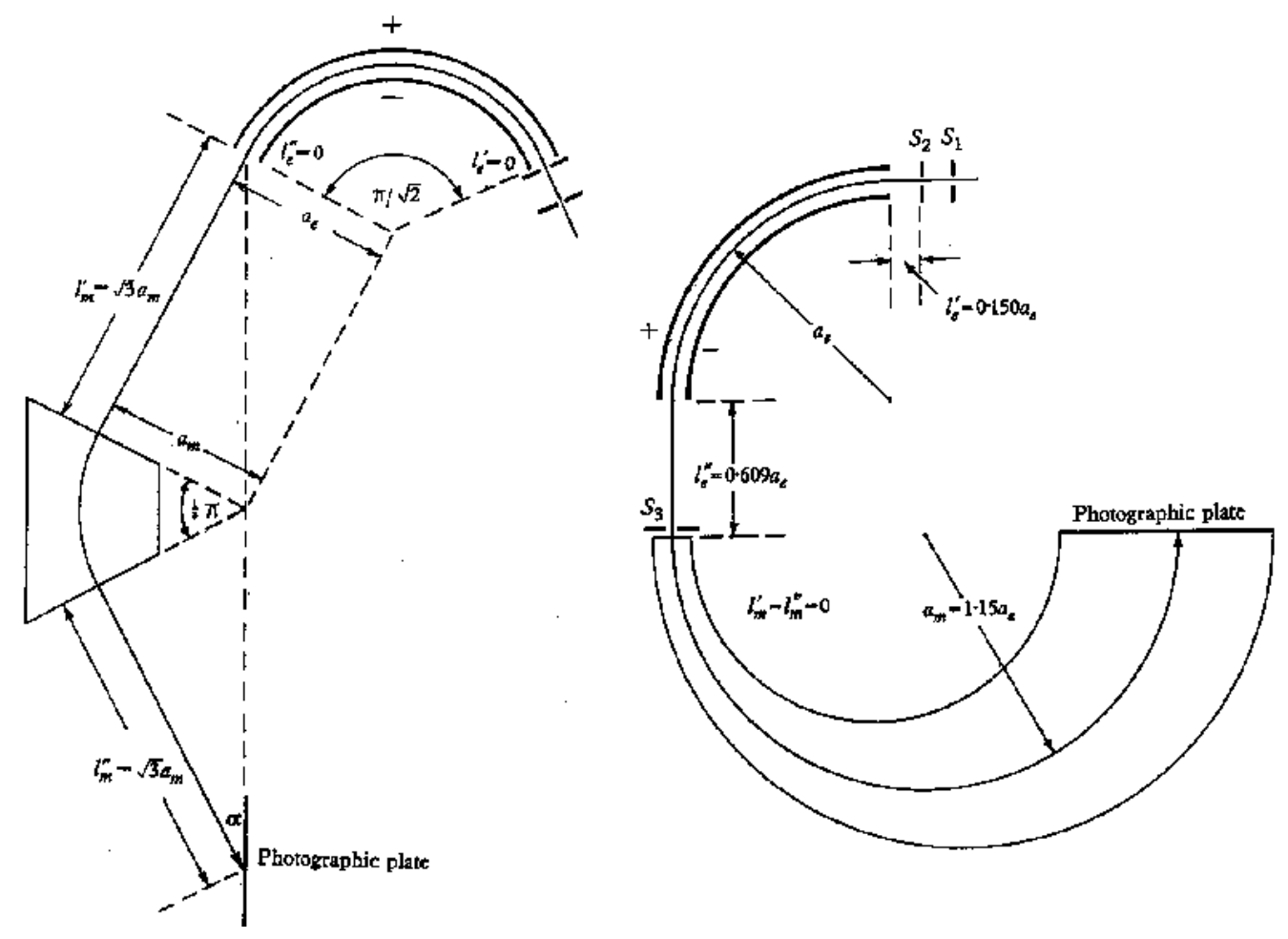

Figure 5: Two examples of double-focusing mass spectrographs: Bainbridge and Jordan's (left); and Dempster's (right). From Ref. [1].

second enlarged version in 1956, $R=75000$.

Another important contribution to nuclear physics was brought by Benjamin G. Hogg and Henry E. Duckworth [13], in 1954, when they discovered nuclear-shape deformation in the rare-earth region after $N=90$, with a Dempster-type double-focusing mass spectrograph, see Fig.8. "The extra stability in the heavier rare-earth region is not adequately explained on a strict one-particle picture". They associated this extra stability with predicted "mixing of configurations".

The interested reader will find more details, and also references to the instruments mentioned above, and for which I gave no citation, in three documents :

i) the book of Francis W. Aston titled "Mass Spectra and Isotopes" [1;

ii) the book of Henry E. Duckworth titled "Mass Spectroscopy" 11, and its update 14];

iii) the article "Atomic Masses: Thomson to Ion Traps" by Aaldert H. Wapstra [15].

\section{Mass spectrometry of unstable nuclides - New spectrometers}

In the early 1970's Robert Klapisch and Catherine Thibault [16] coupled, for the first time, a classical mass spectrometer to an accelerator (Fig.9), the PS at CERN, to measure the masses of unstable species. They discovered that the magicity at $N=20$ 


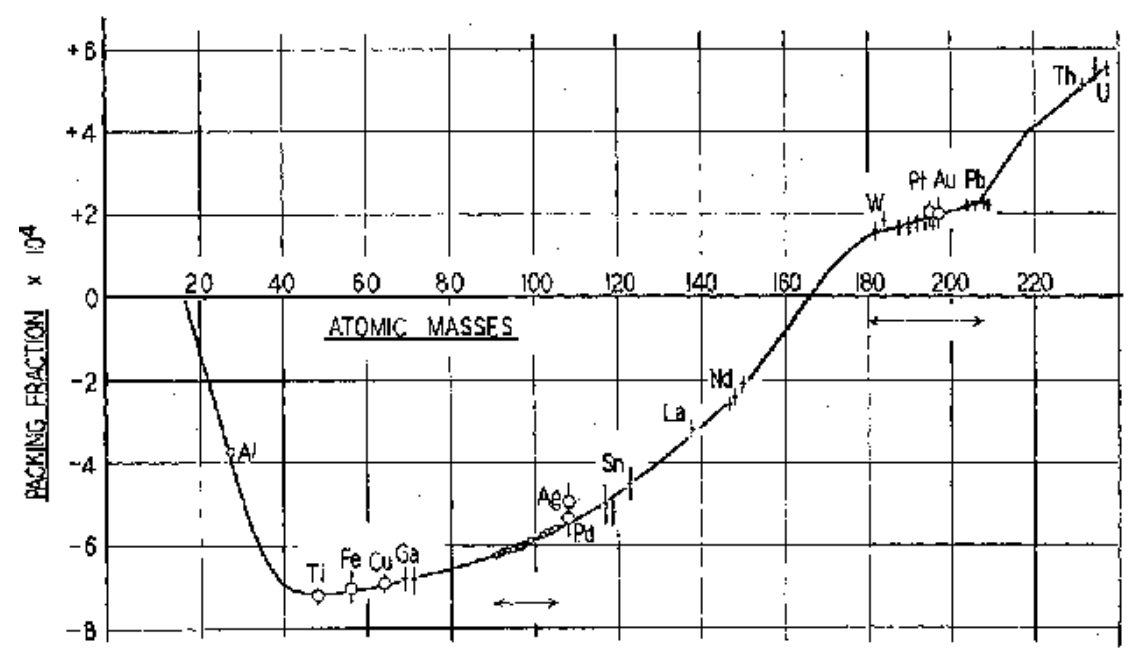

Figure 6: Dempster's packing fraction curve (1938). From Ref. 12].

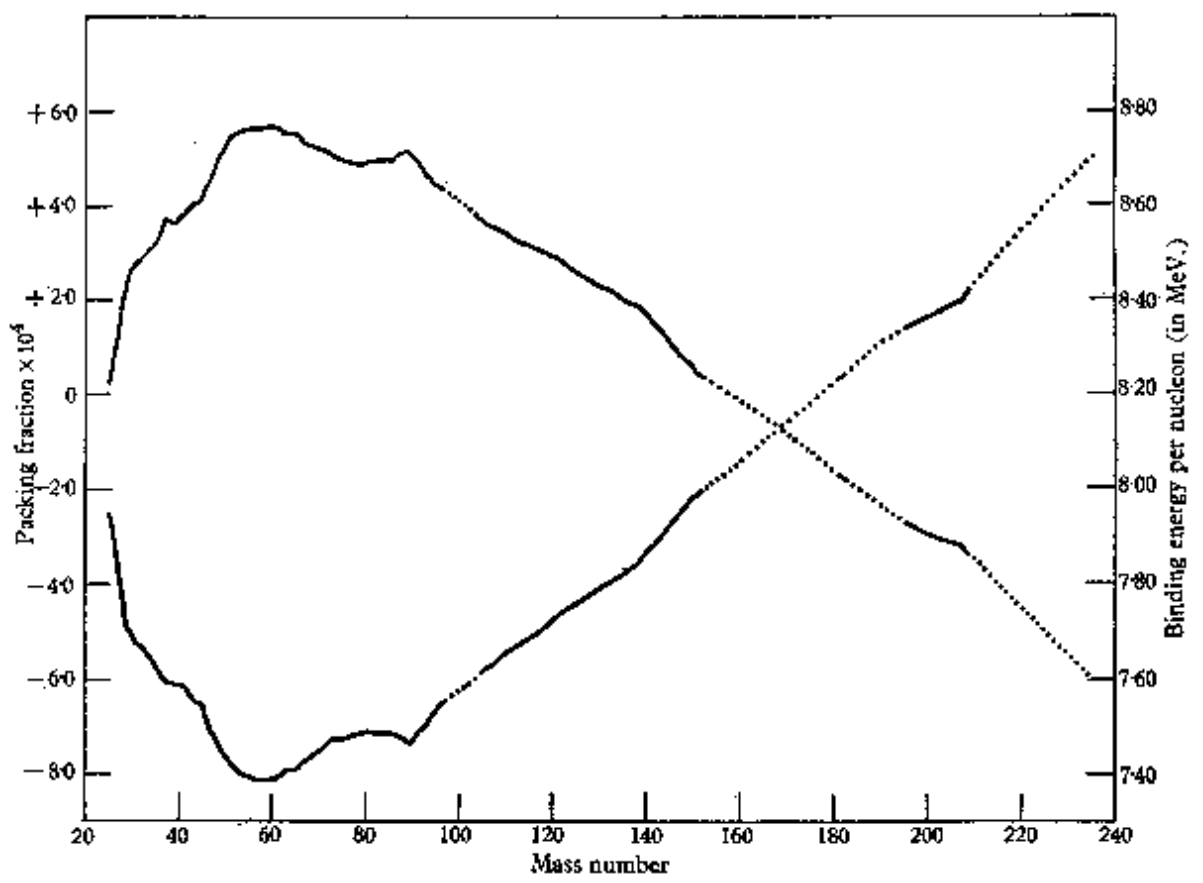

Figure 7: Duckworth's packing fraction curve (1958). The second curve with scale on the right is for the binding energy per nucleon. The now well known structures are clearly visible ( $A=90$ for magic $N=50, A=140$ for magic $N=82$, and $A=208$ for ${ }_{82}^{208} \mathrm{~Pb}_{126}$ ). From Ref. 11. 


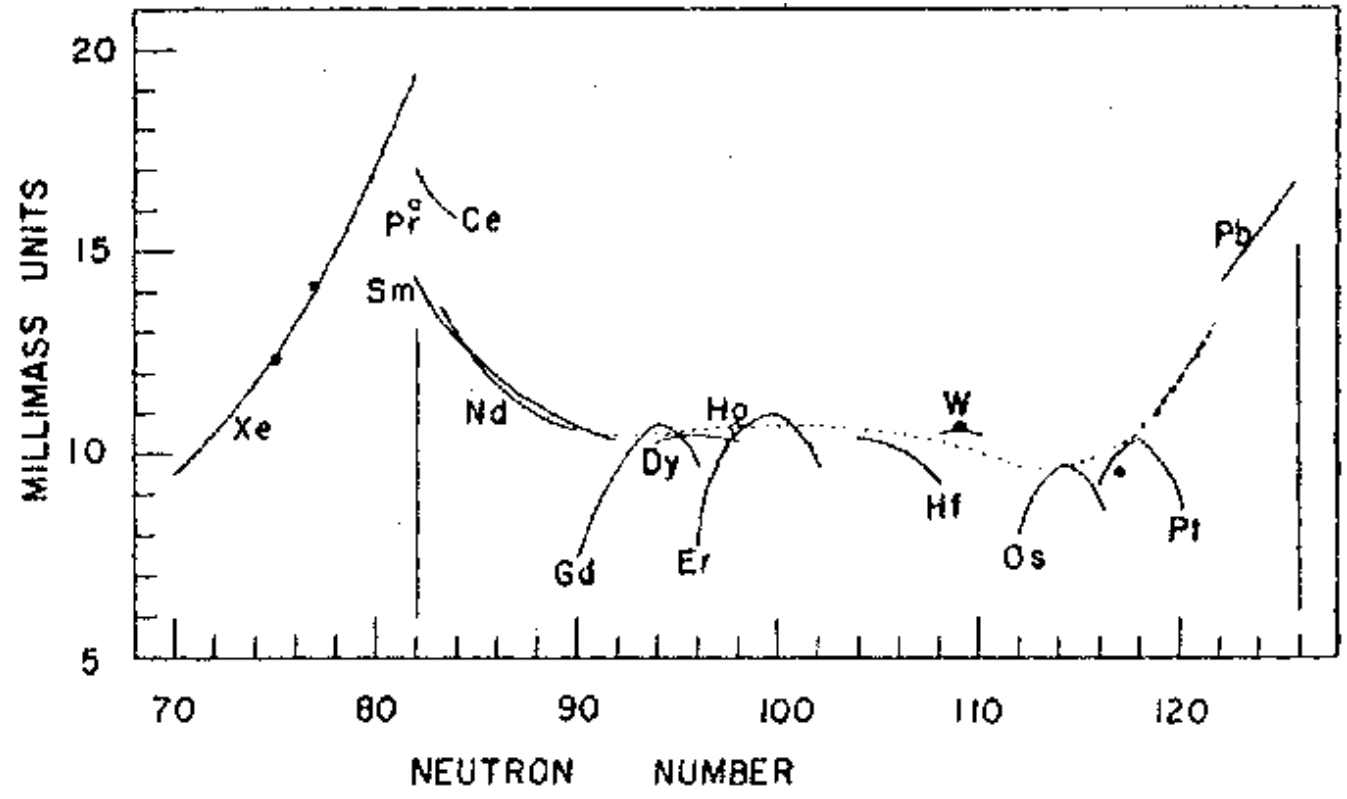

Figure 8: Plot of experimental deviation from a semi-empirical mass formula (expressed in milli-mass unit). The solid curves are the best fits for points belonging to even- $A$ even- $Z$ nuclei. From Ref. [13].

disappeared for $\mathrm{Na}$ isotopes. For my $\mathrm{PhD}$ thesis [17, in 1981 in the same group, I coupled a Mattauch-Herzog double-focusing spectrometer to IsOLDE-II, also at CERN (Fig.10), obtaining a resolving power of $R=60000$. However, for the faint beams of radioactive species, I widened the slits and achieved a typical value of $R=20000$ during operation. I found in the series of Rubidium isotopes a subshell closure at $N=56$ and a deformation starting at $N=60$.

In principle, history stops where the historian's own history starts. Even more so when the historian is an actor in the considered domain. But let me mention some of the most important steps that happened since then.

Around 1980, Jürgen Kluge [18 had the great idea to exploit the fantastic resolving power of Penning traps ${ }^{14}$ in order to perform nuclidic mass measurements. Before the end of that decade, he and his group effectively obtained masses of unstable nuclear species with unprecedented precisions [20].

In the late 1980's, Dave Pritchard [21] at MIT built a Penning trap for stable species, with which the incredible relative precision of $10^{-10}$ for masses up to $A=40$ was obtained. Almost simultaneously Gerald Gabrielse 22] built a trap that he installed at CERN close to an antiproton factory, to compare the mass of the proton with that

\footnotetext{
${ }^{14}$ The "Penning trap", first designed in 1949, is an instrument that combines an electric and a magnetic field in such a way that ions are trapped in a very small volume. Its development lead to one of the most drastic change in the landscape of mass spectrometry. The device received its name after Frans M. Penning who, in 1936, 'trapped' electrons in a magnetic field to increase their path in vacuum and thus increase the sensitivity of ionization vacuum gauges. (See the more detailed history of ion traps in Ref. [19]).
} 


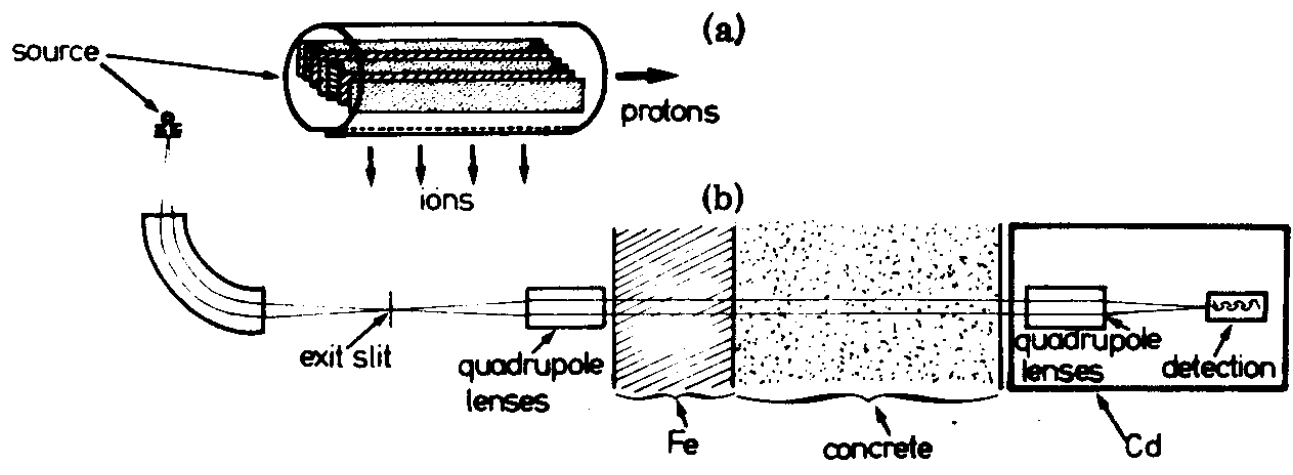

Figure 9: Klapisch and Thibault's mass spectrometer coupled to the PS accelerator at CERN. (a) enlargement of the target, made of graphite foils coated with uranium, which is also the ion source of the spectrometer. (b) schematic lay-out of the spectrometer. Radioactive ions are produced by the proton beam impinging on uranium. They are focused in the ion source optics, enter the magnet, then pass through the exit slit. They are then transported through iron, concrete and cadmium shielding to a station where they are refocused and counted one by one by a high gain ion multiplier. From Ref. [16].
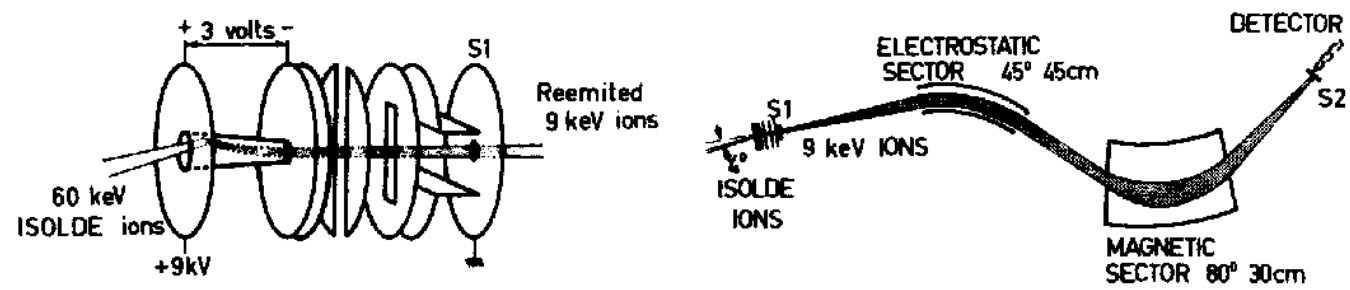

Figure 10: Diagram of Audi's double-focusing Mattauch-Herzog type mass spectrometer coupled to the mass separator 'on-line', IsOLDE-II. Left: the $60 \mathrm{keV}$ IsOLDE ions are stopped in the first atomic layers inside a tantalum tube heated by a 3 volts DC current. The atoms diffuse out of the tantalum matrix, are reionized, accelerated to $9 \mathrm{keV}$ and focused on the entrance slit of the spectrometer $S 1$. Right: the radioactive ions travel through the spectrometer to the exit slit $S 2$ and are detected by an ion multiplier. From Ref. [17].

of the antiproton with a precision of $9 \times 10^{-11}$, which is remarkable for a species so difficult to isolate in our matter-dominated universe ${ }^{15}$. He could thus prove the CPT conservation for the masses at this level of precision.

Today, Penning traps dominate the landscape of mass spectrometry, not only for mass measurements, but in almost all fields using mass spectrometers. Just to be complete, let us mention the other important developments in mass spectrometry: the time-of-flight mass spectrometer of A.E. Cameron and and D.F. Eggers (1948), the radio-frequency mass spectrometer of Lincoln Smith (1960); and the so-called "Schottky

\footnotetext{
${ }^{15}$ The antiproton is expected to live as long as the proton. However, it annihilates in the presence of matter.
} 
mass spectrometry" (1994), at GSI using the EsR storage ring as a spectrometer ${ }^{16}$.

In parallel to the development of mass spectrometry, important development in ion sources extended the study to all types of elements. One can mention here the work of Alfred O. Nier, in 1940, on electron-impact ion sources ${ }^{17}$.

Fig.11 illustrates the increase of precision obtained in the last 70 years in the determination of the masses of ${ }^{14} \mathrm{~N}$ and ${ }^{28} \mathrm{Si}$. Strikingly, on the average, one order of magnitude has been gained every 10 years, from 400 and $600 \mu \mathrm{u}$ respectively in 1937 to 0.6 and $2 \mathrm{nu}$ in 2003. The precision in the mass of ${ }^{28} \mathrm{Si}$ is important in view of the redefinition of the last non-microscopic SI unit, the kilogram. One can remark a seemingly saturation of the precision in ${ }^{28} \mathrm{Si}$ after 1970 and for almost 20 years (the "plateau" at $0.7 \mathrm{keV}$ ), and a very rapid recovery in 1995 due to the MIT Penning trap work. Extrapolating the global tendency, one might expect a precision of $10^{-11}$ or $0.2 \mathrm{nu}$ in 2005 , and that we will reach a precision of $10^{-12}$ in 2015 . As a matter of fact a paper just published [23] shows that ratio of masses could be determined with a precision of $7 \times 10^{-12}$, opening the possibilities to determine the mass of ${ }^{28} \mathrm{Si}$ with a precision close to this number.
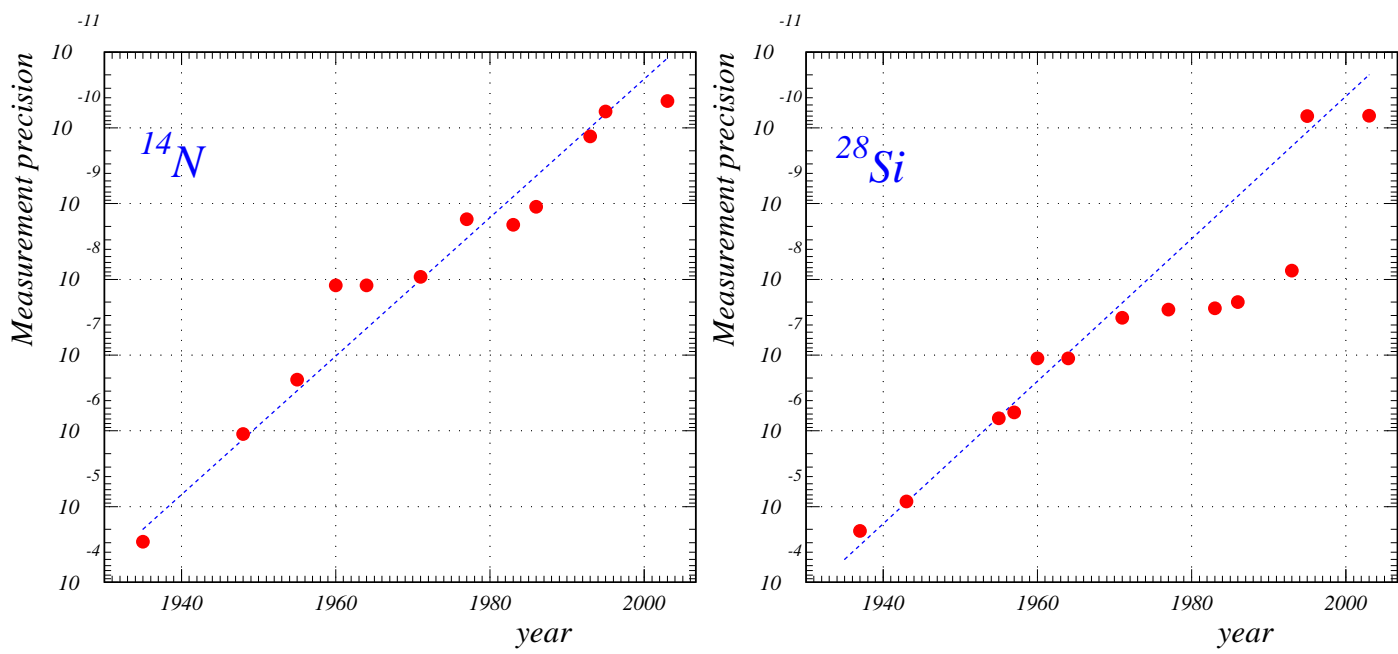

Figure 11: Evolution in the precision with which the masses of ${ }^{14} \mathrm{~N}$ and ${ }^{28} \mathrm{Si}$ have been known between 1937 and 2003. Our knowledge of these masses has increased by one order of magnitude per decade. The values used to plot this figure are from mass tables, respectively for year 1935 from Ref. 24, 1937 from Ref. 25], 1943 from [26], 1948 [27, 1955 [28, 1957 [29], 1960 [30], 1964 [31, 1971 [32, 1977 [33], 1983 34, 1986 35], 1993 [36, 1995 [37] and 2003 [8.

\footnotetext{
${ }^{16}$ named after Walter Schottky, 1886-1976, a German physicist who discovered the random noise due to the irregular arrival of electrons at the anode of thermionic tubes that is called "shot noise". In Schottky mass spectrometry, ions circulate in a ring. At each turn, a detector consisting of pick-up electrodes (called "Schottky" electrodes), records their time of passing. The rotation frequencies are thus determined. Therefore, strictly speaking, Schottky is not the name of a spectrograph, but that of a detection device, the spectrograph being the storage ring.

17 "The construction of these instruments (spectrometers built in the mid 1950's) was concurrent with new developments in ion optics, where the study of the effects of second order aberrations and of fringing fields were being pursued vigorously. ... The important development of vacuum and electronic technology during the WWII years led to enormous improvements in the post-war instruments." [10].
} 


\section{A short history of the mass unit}

A mass measured by mass spectrometry is determined as an inertial mass, from its movement characteristics in an electro-magnetic field. More exactly, the quantity measured "directly" is the ratio of the mass of the nuclide of interest to a well known mass. The result is then expressed in 'unified atomic mass' $(u)^{18}$, or its sub-unit, $\mu \mathrm{u}$.

Mass measurements can also be obtained "indirectly" as differences in energy between neighboring nuclides, either by measuring a decay energy or a reaction energy. An energy relation is thus established between the mass we want to determine and a well known nuclidic mass. This energy relation is then expressed in electronvolts $(\mathrm{eV})$.

Two units are thus used in atomic mass measurements. We shall examine them separately and discuss how they are related.

The mass unit is defined, since 1960 , by $1 \mathrm{u}=\mathcal{M}\left({ }^{12} \mathrm{C}\right) / 12$, as one twelfth of the mass of one free atom of carbon-12 in its atomic and nuclear ground-state. Before 1960, as Wapstra once told me, two mass units were defined: the physical one $\mathcal{M}\left({ }^{16} \mathrm{O}\right) / 16$, and the chemical one which represented one sixteenth of the average mass of a standard mixture of the three stable isotopes of oxygen ${ }^{19}$. Physicists could not convince the chemists to drop their unit; "The change would mean millions of dollars in the sale of all chemical substances", said the chemists, which is indeed true! Joseph H.E. Mattauch, the American chemist Truman P. Kohman and Aaldert H. Wapstra 38 then calculated that, if $\mathcal{M}\left({ }^{12} \mathrm{C}\right) / 12$ was chosen, the change would be ten times smaller for chemists, and in the opposite direction... That lead to unification; ' $u$ ' stands therefore, officially, for 'unified mass unit'! To be complete, let us mention that the chemicalmass spectrometry community (e.g. bio-chemistry, polymer chemistry,...) often use the dalton ${ }^{20}$ (symbol Da), which, whatever is claimed, serves actually to determine the number of nucleons in a molecule, with not too much concern about the exact value of the obtained mass compared to ${ }^{12} \mathrm{C}$. It is thus not strictly the same as ' $\mathrm{u}$ '. As a matter of fact, some attempts were made to determine atomic masses with equipment used in chemistry. However, the values obtained [39, 40, 41. appeared later to be at strong variance compared to modern results.

The energy unit is the electronvolt. Until recently, the relative precision in $M-A$ expressed in $\mathrm{keV}$ was, for several nuclides, less good than the same quantity expressed in mass units. The choice of the volt for the energy unit (the electronvolt) is not evident. One might expect the use of the international volt $\mathrm{V}$, but one can also

\footnotetext{
${ }^{18}$ Quite often people write erroneously 'a.m.u' or 'amu' instead of 'u'.

${ }^{19}$ R.C. Barber's comment 10]: "The chemists used 1/16 of the mass of oxygen as the mass unit, beginning with very early work, back when they literally weighed the components in chemical reactions. As soon as Aston saw the evidence for isotopes of oxygen he realized that the definition, based on a 'natural' abundance ratio for the three isotopes, was inherently imprecise. He defined all of his masses relative to ${ }^{16} \mathrm{O}$. This situation went on for decades. The chemists were untroubled by the slight difference, while the physicists were content with the Aston definition for a long time. However, with increasing precision, it was realized that mass spectroscopic comparisons were always referred to a 'standard', known hydrocarbon molecule that always involved ${ }^{12} \mathrm{C}$. To convert from the defined standard ${ }^{16} \mathrm{O}$ to ${ }^{12} \mathrm{C}$, one had to study the ${ }^{12} \mathrm{C}^{1} \mathrm{H}_{4}-{ }^{16} \mathrm{O}$ doublet that was not particularly well known. If one changed to the ${ }^{12} \mathrm{C}$ definition, there was an immediate 'free' improvement in precision."

${ }^{20}$ named after John Dalton, 1766-1844, a British scientist who first speculated that elements combine in proportions following simple laws, and was the first to create a table of (very approximate) atomic weights.
} 
choose the standard volt $\mathrm{V}_{90}$ as maintained in national laboratories for standards and defined by adopting an exact value for the constant $(2 e / h)$ in the relation between frequency and voltage in the Josephson effect. In the 1999 table of standards 42]: $2 e / h=483597.9$ (exact) $\mathrm{GHz} / \mathrm{V}_{90}$. An analysis by E.Richard Cohen and Aaldert H. Wapstra [4] showed that all precision measurements of reaction and decay energies were calibrated in such a way that they can be more accurately expressed in standard volt. Also, the precision in the conversion factor between mass unit and standard volt, $\mathrm{V}_{90}$, is more accurate than the conversion of the mass unit to the international volt $\mathrm{V}$ :

$$
\begin{aligned}
& 1 \mathrm{u}=931494.0090 \pm 0.0071 \mathrm{keV}_{90} \\
& 1 \mathrm{u}=931494.013 \pm 0.037 \mathrm{keV}
\end{aligned}
$$

This has not always been the case. In the early days of the evaluation of masses, two independent evaluations and adjustments were often performed, separately for reaction and decay data, and for mass spectrometric measurements. Comparing the two allowed one to derive a value for the conversion factor, which could then be compared to the one derived by other, more precise, methods 42 . The present computer program for the least-squares fit of the mass adjustment still contains an option that allows this conversion factor to be a free parameter.

The reader will find more information on the energy unit, and also some historical facts about the electronvolt, in the AME2003 44, page 134.

\section{The history of the evaluation of atomic masses}

It was felt very early that establishing lists of properties for nuclei was not only useful, but necessary. Several collections were thus published. Below is a list of the atomic mass compilations. The first one, to my knowledge, in which data from mass spectrometry and nuclear reaction and decay data were combined, is the 1937 table of Milton Stanley Livingston and Hans Albrecht Bethe [25.

$\begin{array}{llll}1935 & \text { H. Bethe } & {[24} & \text { evaluation and table }{ }^{1} n-{ }^{17} \mathrm{O} \\ 1937 & \text { M.S. Livingston and H.A. Bethe } & {[25} & \text { combined evaluation: energies + masses } \\ 1943 & \text { S. Flügge and J.H.E. Mattauch } & {[26} & \\ 1944 & \text { G. Seaborg } & {[45} & \\ 1946 & \text { G. Seaborg } & {[46} & \text { "The Plutonium project table" } \\ 1946 & \text { J. Suruque } & {[47} & \\ 1948 & \text { A.H. Wapstra } & {[27} & \text { "Table of atomic nuclei" } \\ 1953 & \text { A.H. Wapstra } & {[48} & A>200\end{array}$

In the early 1950's it was found that the many relations (direct and indirect) overdetermined the mass value of many nuclides. Aaldert H. Wapstra established a procedure using a least-squares method to solve the problem of overdetermination. One of the side-benefits of the overdetermination is to get a check of the consistency among the various results. The first table of atomic masses using this method is dated 1955 [28].

Since then, A.H. Wapstra has carried on the evaluation of the experimental masses of nuclei - to be more precise their atomic masses - in what we call the Atomic Mass Evaluation (AME) with various students or collaborators, until I joined him in 1981. 
We published together every 10 years since then (1983, 1993 and 2003) a complete set of masses and of the data from which they are deduced.

The list below gives the main "modern" evaluations of atomic masses following the general lines as first defined in Ref. [28, well described in Ref. 49] and slightly refined since then (see the most recent and most complete of those in the AmE2003 44]):

\begin{tabular}{|c|c|c|c|}
\hline 1955 & A.H. Wapstra and J.R. Huizenga & 28 & "Isotopic masses" \\
\hline 1956 & J. Mattauch et al & 50 & "The masses of light nuclides \\
\hline 1957 & J.H.E. Mattauch and F. Everling & 29 & "Masses of atoms of $A<40$ \\
\hline 1960 & F. Everling et al & sou & "Relative nuclidic masses" \\
\hline 1962 & L.A. König et al & 51 & "1961 nuclidic mass table" \\
\hline 1965 & J.H.E. Mattauch et al & 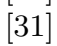 & "1964 atomic mass table" \\
\hline 1971 & A.H. Wapstra and M.B. Gove & 3 & "The 1971 atomic mass evaluation" \\
\hline 1977 & A.H. Wapstra and K. Bos & $x$ & "The 1977 atomic mass evaluation" \\
\hline 1985 & A.H. Wapstra and G. Audi & 3 & "The 1983 atomic mass evaluation" \\
\hline 1993 & G. Audi and A.H. Wapstra & 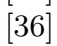 & "The 1993 atomic mass evaluation" \\
\hline 2003 & G. Audi et al & & "The AмE2003 atomic mass evaluation" \\
\hline
\end{tabular}

With the development of accelerators and the production of an increasing number of unstable species, excited nuclear states were increasingly populated. Those with half-lives long compared to typical electromagnetic transitions (femtoseconds fs to picoseconds ps) started to play a rôle in the measurement of decay energies. These long-lived excited states are called the excited isomers ${ }^{21}$. In many measurements of decay energies, it is not well established if the emitting level from the mother nucleus is the ground-state or an isomeric level. Often simultaneous measurement of the halflife or of the spin (through the transition probabilities) removes the ambiguity. The problem, however, becomes worse in mass spectrometry where the measurement may only yield one line for a mixture of closely lying isomers. The interpretation is then difficult $^{22}$. Only in favorable cases, corrections could be estimated.

As a consequence, not only had the AmE to handle several isomers for each nuclidic species, but also one needed a unique and consistent description of the various isomers that were involved in the AmE. Therefore the NuBASE database was created in 1993 and published since then in 1997 and 2003 [53, the latter in complete synchronization with the AME2003.

\section{Conclusion}

"The history of nuclear masses is almost as old as that of nuclear physics itself." was the first sentence of the introduction. The conclusion can complement this statement in that the history of nuclear masses and the history of its most important contributor, namely mass spectrometry, have continuously fed nuclear physics with major discoveries. Each

\footnotetext{
${ }^{21}$ Here is another example of common misuse of terms. Strictly, we should distinguish ground-state isomer and excited isomers, as chemists distinguish left-handed and right-handed ones. Often people call 'isomer' the excited one. (the Merriam-Webster says "isomerism: the relation of two or more nuclides with the same mass numbers and atomic numbers but different energy states and rates of radioactive decay").

${ }^{22}$ Then, it is fully worthy to repeat the experiment with increased resolving power, even at the cost of lower counting rate and decreased precision. The final result will be more accurate. See the remarkable example of $\mathrm{Hg}$ isomers in Ref. [52].
} 
progress in building new spectrometers, increasing resolving power or sensitivity or both, has led, as shown in this paper, to important new physics. We have seen some of them: the nature of channel rays; the discovery of isotopism; the restoration of the whole number rule; the explanation of the age of the Sun; the discovery of mass defect and the experimental proof of the equivalence of mass and energy; the discovery of the magic numbers; the discovery of deformations; the discovery of a subshell closure; the discovery that magic numbers might disappear; ... Much more could be said on recent breakthroughs in our understanding of physics brought about by mass measurements, the history of which is still to be written.

In parallel to this history, and strongly related to it, is the history of the atomic mass evaluation that establishes and ascertains our confidence in the measured masses.

\section{Acknowledgements}

I would like to thank Aaldert H. Wapstra who, since 1981, made me discover the fascinating field of mass evaluation. Doing this job makes us see almost all aspects of the nuclear physics problems and many also beyond. Often, we have the privilege to be the first ones to see new phenomena. Therefore, thank you Aaldert for having let me enter this beautiful domain. Now, we would both be so happy to have someone willing to share with us the same passion.

I want to thank Jürgen Kluge, to whom this paper is dedicated in honor of his 65 th anniversary. Jürgen invited me, in 1987, just after the first run with the Penning trap at ISOLDE, to join his team and enjoy the flourishing program and the tremendous amount of data he and his group obtained with this splendid spectrometer.

I wish also to thank those who helped me in this work: first of all Lutz Schweikhard for asking me to write this piece of history and for several very useful suggestions; and R.C. Barber, F. Everling, C. Gaulard, D. Lunney, C. Thibault and A.H. Wapstra for reading, and making corrections and suggestions to improve the manuscript.

\section{References}

[1] F.W. Aston, "Mass Spectra and Isotopes", Second edition, Edward Arnold \& Co., 1942.

[2] F.W. Aston, Nature 105 (1920) 617.

[3] A. S. Eddington, Nature 106 (1920) 14; "The Internal Constitution of the Stars", Presidential Address to Section A of the British Association at Cardiff, on 24th August 1920, The Observatory XLIII (October 1920) No. 557.

[4] W.M. Elsasser, a) J.Phys. 4 (1933) 549; b) J.Phys. 5 (1934) 389; c) J.Phys. 5 (1934) 635.

[5] W.M. Elsasser, C.R.Acad.Sc. 199 (1934) 46.

[6] M. Goeppert-Mayer, Nobel Lecture, December 12, 1963, Elsevier Publishing Company, Amsterdam, 1972, http://nobelprize.org/physics/laureates/1963/mayer-lecture.html

[7] M. Goeppert-Mayer, Phys. Rev. 74 (1948) 235.

[8] G. Audi, A.H. Wapstra and C. Thibault, Nucl. Phys. A 729 (2003) 337.

[9] http://www.nndc.bnl.gov/amdc/masstables/Ame2003/graphs2003/ or http://amdc.in2p3.fr/masstables/Ame2003/graphs2003/filel.html

[10] R.C. Barber, Private Communication, January 2006. 
[11] H.E. Duckworth, "Mass Spectroscopy", First edition, Cambridge University Press, 1958. See also the article of R.C. Barber and K.S. Sharma, Nucl. Instrum. Meth. B 204 (2003) 460.

[12] A.J. Dempster, Phys. Rev. 53 (1938) 869.

[13] B.G. Hogg and H.E. Duckworth, Phys. Rev. 91 (1953) 1289; Can. J. Phys. 32 (1954) 65; and R.C. Barber, H.E. Duckworth, B.G. Hogg, J.D. Macdougall, W. McLatchie and P.Van Rookhuyzen, Phys. Rev. Lett. 12 (1964) 597.

[14] H.E. Duckworth, R.C. Barber, V.S. Venkatasubramanian, "Mass Spectroscopy", Second edition, Cambridge University Press, 1986.

[15] A.H. Wapstra, Phys. Scripta T 59 (1995) 65.

[16] R. Klapisch, R. Prieels, C. Thibault, A.M. Poskanzer, C. Rigaud and E. Roeckl, Phys. Rev. Lett. 31 (1973) 118.

[17] G. Audi, "Mesures de masses atomiques de noyaux exotiques", Doctoral Thesis \#2486, Université d'Orsay, 1981; http://www.nndc.bnl.gov/amdc/experimental/th-audi.pdf

[18] P. Dabkiewicz, H. Kalinowski, F. Kern, H.-J. Kluge, L. Schweikhard, H. Schnatz, H. Stürmer and R.B. Moore, Proc. 7th Int. Conf. Atomic Masses and Fundamental Constants AMCO-7, 1984, p.684.

[19] M.H. Holzscheiter, Phys. Scripta T 59 (1995) 69.

[20] H. Stolzenberg, St. Becker, G. Bollen, F. Kern, H.-J. Kluge, Th. Otto, G. Savard, L. Schweikhard, G. Audi and R.B.Moore, Phys. Rev. Lett. 65 (1990) 3104.

[21] E.A. Cornell, R.M. Weisskoff, K.R. Boyce, R.W. Flanagan, Jr., G.P. Lafyatis and D.E. Pritchard, Phys. Rev. Lett. 63 (1989) 1674.

[22] G. Gabrielse, X. Fei, K. Helmerson, S.L. Rolston, R. Tjoelker, T.A. Trainor, H. Kalinowsky, J. Haas and W. Kells, Phys. Rev. Lett. 57 (1986) 2504.

[23] S. Rainville, J.K. Thompson, E.G. Myers, J.M. Brown, M.S. Dewey, E.G. Kessler,Jr., R.D. Deslattes, H.G. Börner, M. Jentschel, P. Mutti and D.E. Pritchard, Nature 48 (2005) 1096.

[24] H. Bethe, Phys. Rev. 47 (1935) 633.

[25] M.S. Livingston and H.A. Bethe, Rev. Mod. Phys 9 (1937) 245.

[26] S. Flügge and J.H.E. Mattauch, Physikal. Zeitschr. 44 (1943) 181 and 391.

[27] A.H. Wapstra, "Table of atomic nuclei", in L. Rosenfeld, "Nuclear Forces", North-Holland, Amsterdam, 1948, p. 497.

[28] A.H. Wapstra, "Isotopic masses I. $A<34$; II. $33<A<202$ ", Physica 21 (1955) 367 and 385; and J.R. Huizenga, "Isotopic masses III. $A>201 "$, Physica 21 (1955) 410.

[29] J. Mattauch and F. Everling, Progr. Nucl. Phys. 6 (1957) 233.

[30] F. Everling, L.A. König, J.H.E. Mattauch and A.H. Wapstra, "Atomic masses of nuclides for A $\leq 70 "$, Nucl. Phys. A 15 (1960) 342; and "Relative nuclidic masses", Nucl. Phys. A 18 (1960) 529 .

[31] J.H.E. Mattauch, W. Thiele and A.H. Wapstra, Nucl. Phys. A 67 (1965) 1.

[32] A.H. Wapstra and M.B. Gove, Nucl.Data.Tables 9 (1971) 267.

[33] A.H. Wapstra and K. Bos, Nucl.Data.Tables 19 (1977) 177.

[34] A.H. Wapstra and G. Audi, Nucl. Phys. A 432 (1985) 1.

[35] A.H. Wapstra, G. Audi and R. Hoekstra, At. Nucl. Data Tables 39 (1988) 281.

[36] G. Audi and A.H. Wapstra, Nucl. Phys. A 565 (1993) 1.

[37] G. Audi and A.H. Wapstra, Nucl. Phys. A 595 (1995) 409.

[38] T.P. Kohman, J.H.E. Mattauch and A.H. Wapstra, J. de Chimie Physique 55 (1958) 393; Science 127 (1958) 1431.

[39] E.N. Nikolaev, Yu.I. Neronov, M.V. Gorshkov and V.L. Talroze, Pisma Zh. Eksp. Teor. Fiz. 39 (1984) 441. 
[40] E. Lippmaa, R. Pikver, E. Suurmaa, J. Past, J. Puskar, I. Koppel and A. Tammik, Phys. Rev. Lett. 54 (1985) 54.

[41] J.T. Meek, W.G. Millen, G.W. Stockton and R.T. Kouzes, Phys. Rev. C 41 (1990) 2921.

[42] P.J. Mohr and B.N. Taylor, J. Phys. Chem. Ref. Data 28 (1999) 1713.

[43] E.R. Cohen and A.H. Wapstra, Nucl. Instrum. Meth. 211 (1983) 153.

[44] A.H. Wapstra, G. Audi and C. Thibault, Nucl. Phys. A 729 (2003) 129.

[45] G. Seaborg, Rev. Mod. Phys. 16 (1944) 1.

[46] G. Seaborg, "The Plutonium project table", Rev. Mod. Phys. 18 (1946) 513.

[47] J. Suruque, J. Physique 8 (1946) 145.

[48] A.H. Wapstra, Thesis, 1953.

[49] F. Everling, L.A. König, J.H.E. Mattauch and A.H. Wapstra, "Adjustment of relative nuclidic masses (I) - A $\leq$ 70", Nucl. Phys. A 25 (1961) 177;

L.A. König, J.H.E. Mattauch and A.H. Wapstra, "Adjustment of relative nuclidic masses (II) $70<A<200 "$, Nucl. Phys. A 28 (1961) 1;

A.H. Wapstra, "Adjustment of relative nuclidic masses (III) - A $\geq 200$ ", Nucl. Phys. A 28 (1961) 29.

[50] J. Mattauch, L. Waldmann, R. Bieri and F. Everling, Ann. Rev. of Nucl. Science 6 (1956) 179.

[51] L.A. König, J.H.E. Mattauch and A.H. Wapstra, Nucl. Phys. A 31 (1962) 18.

[52] S. Schwarz, F. Ames, G. Audi, D. Beck, G. Bollen, C. De Coster, J. Dilling, O. Engels, R. Fossion, J. -E. Garcia Ramos, S. Henry, F. Herfurth, K. Heyde, A. Kellerbauer, H. -J. Kluge, A. Kohl, E. Lamour, D. Lunney, I. Martel, R. B. Moore, M. Oinonen, H. Raimbault-Hartmann, C. Scheidenberger, G. Sikler, J. Szerypo and C. Weber, Nucl. Phys. A 693 (2001) 533.

[53] G. Audi, O. Bersillon, J. Blachot and A.H. Wapstra, Nucl. Phys. A 729 (2003) 3. 